\title{
Pediatric Malignant Mediastinal Masses
}

\author{
Rahat-ul-Ain Kashif, Mahwish Faizan and Saadia Anwar
}

\begin{abstract}
Objective: To describe the clinical spectrum and outcome-associated variables of pediatric malignant mediastinal masses in a resource-limited setting.

Study Design: Descriptive study.

Place and Duration of Study: Department of Pediatric Hematology-Oncology, The Children's Hospital, Lahore, from October 2016 to November 2017.

Methodology: Children with malignant mediastinal masses were enrolled. The variables studied were median age at presentation, gender distribution, immunisation status, socio-economic background, causes of delayed presentation, presenting complaints, complications of disease, methods for mass biopsy, final diagnosis, staging, and outcome of the disease. Results were described in terms of descriptive statistics.

Results: The median age at diagnosis was 7.5 years with male-to-female ratio of 2:1. The commonest presenting complaint was fever $(82 \%)$, respiratory distress $(58 \%)$, and lymphadenopathy $(51 \%)$. Seventy-eight percent patients belonged to lower socio-economic class. Eighty-six percent of patients had delayed presentation to the tertiary care hospital and the most common reason was delayed diagnosis by the medical professionals (49\%). Fifty-one percent patients had weight-for-age less than 5th percentile. Common complications were airway obstruction (35\%), pericardial effusion (19.6\%), superior vena cava syndrome and gross pleural effusion (13.7\% each). Commonest diagnosis was T-cell acute lymphoblastic leukemia (35\%) followed by lymphoblastic lymphoma and Hodgkin's lymphoma (15.7\% each). Fourtyfive percent patients expired, $2 \%$ defaulted treatment and $5.9 \%$ completed treatment; $25 \%$ patients were under treatment, $3.9 \%$ patients had progressive disease while outcome of $17.6 \%$ of patients could not be known. The most significant factor associated with the outcome primary diagnosis $(p<0.001)$, delayed presentation $(p=0.007)$ and educational status of the family.

Conclusion: The pattern of clinical presentation, complications and diagnoses of pediatric malignant mediastinal masses showed some variation from the already reported. Low literacy rate and delay in presentation are common and contribute significantly to the poor outcome.
\end{abstract}

Key Words: Mediastinal masses, Primary malignant mediastinal tumors, Pediatric mediastinal malignancies.

\section{INTRODUCTION}

Primary mediastinal malignancies are rare in children and can originate from any mediastinal tissue such as thymic, neurogenic, lymphatic, germinal, or mesenchymal. ${ }^{1}$ There is high incidence of asymptomatic presentation of a mediastinal mass; therefore, the diagnosis and management of a mediastinal mass in children may be challenging. ${ }^{1}$ The signs and symptoms of mediastinal tumors depend on the site, size, and involvement of the adjacent organs. Mediastinal masses in children comprise a range of lesions that have their origin in the embryonic tissues. ${ }^{2}$ They may vary from benign cysts to very aggressive malignant lesions and are responsible for significant morbidity and mortality. ${ }^{3}$

Department of Pediatric Hematology-Oncology and Bone Marrow Transplant, The Children's Hospital and Institute of Child Health, Lahore, Pakistan

Correspondence: Dr. Rahat-ul-Ain Kashif, Department of Pediatric Hematology-Oncology and Bone Marrow Transplant, The Children's Hospital and Institute of Child Health,

Ferozepur Road, Lahore, Pakistan

E-mail:dr.rkashif@yahoo.com

Received: March 10, 2018; Accepted: September 05, 2018
The clinical manifestations of these lesions are nonspecific and, due to the fact that the mass expands within a limited area, compression of adjacent structures is generally seen.2,3 Respiratory tract involvement may present with dyspnea, orthopnea, or pleuritic chest pain; while those with cardiovascular involvement may present with syncope, shortness of breath, and facial swelling. ${ }^{4}$ Early recognition of these life-threatening clinical signs and timely intervention is imperative to improve the outcome.5,6

Lymphomas are the most common etiology of a mediastinal mass in the pediatric age group, accounting for $46-56 \%$ of all mediastinal masses. ${ }^{7}$ Mushtaq et al. in a retrospective study on 32 patients showed that most common diagnosis was lymphoma $(72 \%)$. T-cell leukemia was identified in $25 \%$ cases and $3.1 \%$ patients had neuroblastoma. The median age at diagnosis was nine years with male-to-female ratio being 2:1.8 Airway compression is a serious problem during anesthesia for mass biospy. ${ }^{9}$ Corticosteroids and radiotherapy before biopsy may improve perioperative risk, but also may adversely affect histological diagnostic accuracy. ${ }^{10}$ Ferrari et al. in a review of 163 children with mediastinal mass stressed the importance of tissue diagnosis before initiation of treatment. ${ }^{11}$ 
The objective of this study was to demonstrate the clinical spectrum and outcome-affecting variables of pediatric malignant mediastinal masses in a resource limited setting.

\section{METHODOLOGY}

It was a descriptive study, conducted from October 2016 to November 2017 in Pediatric Hematology-Oncology Department at The Children's Hospital and Institute of Child Health, Lahore, Pakistan with evidence of a mediastinal mass as per operational definition and were fulfilling the inclusion criteria, i.e. children of age group from one month to 16 years with suspected malignant mediastinal mass and suggestive imaging study, while the cases of relapsed malignancies and histology proven non-malignant masses were excluded.

Sampling type was non-probability and purposive type. Approval from Ethical Committee was taken. After taking an informed consent from the parents, the relevant clinical information were collected and noted on the proforma. The variables studied were median age at presentation, gender distribution, immunisation status, socio-economic background, causes of delayed presentation, presenting complaints, complications of disease, methods for mass biopsy, final diagnosis, staging, and outcome of the disease.

All the data were entered and analysed in SPSS version 25.

\section{RESULTS}

There were 51 patients who presented with a suspected malignant mediastinal mass. Male patients were predominant $(70 \%)$, while $30 \%$ patients were female with male-to-female ratio being 2:1. The median age at diagnosis was 7.5 years. Among female patients, almost equal proportion of patients presented in all age groups ranging from 1 to 15 years; while among male patients the predominant age group at presentation was 6-10 years, in which $50 \%$ of the male patients presented. Twenty-eight percent $(28 \%)$ and $22 \%$ of male patients presented in the age group of 11-15 years and 1-5 years, respectively. Majority of the patients (92\%) belonged to Punjab province while $4 \%$ presented from Azad Kashmir province and $2 \%$ patients were from Khyber Pakhtun Khawah province. One patient $(2 \%)$ presented from Afghanistan.

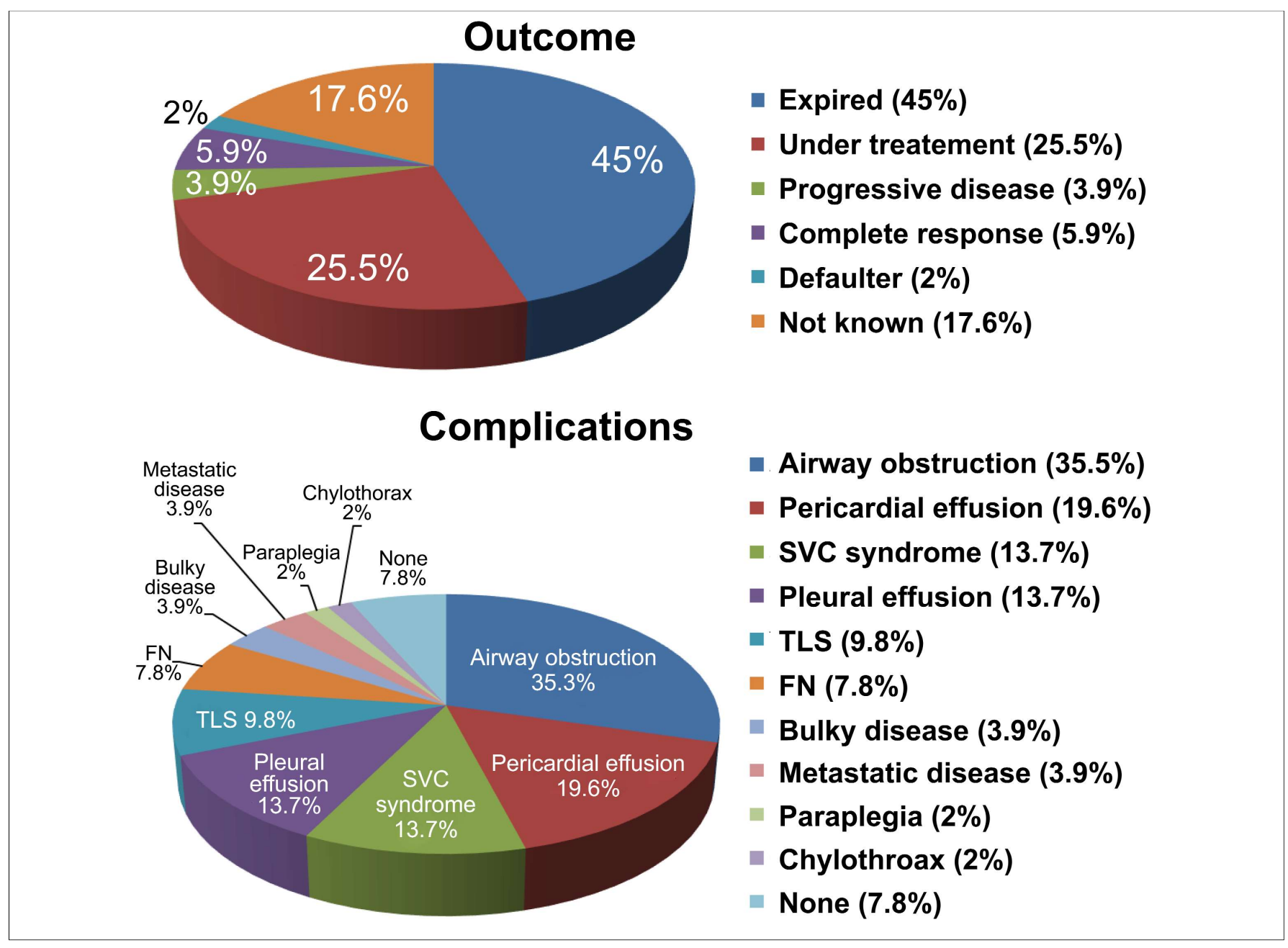

Figure 1: Percentage of complications and outcomes of patients with malignant mediastinal masses. 
Table I: List of presenting complaints in children with malignant mediastinal masses.

\begin{tabular}{l|c|c}
\hline Presenting complaints & Number of patients & $\begin{array}{c}\text { Percentage (\%) } \\
\text { Total number (n)=51 }\end{array}$ \\
\hline Fever & 42 & $82 \%$ \\
Respiratory distress & 30 & $58 \%$ \\
Lymphadenopathy & 26 & $51 \%$ \\
Cough & 23 & $45 \%$ \\
Pale complexion & 20 & $39 \%$ \\
Weight loss & 17 & $33 \%$ \\
Fatigue & 14 & $27 \%$ \\
Orthopnea & 13 & $25 \%$ \\
Bleed & 11 & $22 \%$ \\
Anorexia & 11 & $22 \%$ \\
Facial puffiness & 11 & $21 \%$ \\
Pedal edema & 3 & $6 \%$ \\
Abdominal mass & 2 & $2 \%$ \\
Neurological symptoms & 1 & $2 \%$ \\
(paraplegia) & 1 & $2 \%$ \\
Chest pain & &
\end{tabular}

Regarding the socio-economic status, $78 \%$ patients belonged to lower socio-economic class (PKR. 4,000 PKR. 25,000 per month), 14\% belonged to the lowermiddle class (PKR. 25,000 - PKR. 65,000 per month), and $8 \%$ from the upper middle class (PKR. 65,000 PKR. 250,000 per month). Majority (39\%) of parents (fathers) were uneducated and only $15.7 \%$ parents were graduates. Rest of the parents $(45 \%)$ took education at various levels of schooling (primary, secondary and matric). Majority of the patients $(96 \%)$ were vaccinated according to the EPI (Expanded Programme on Immunisation) Pakistan. None of the patients was asymptomatic on presentation. The frequency of various presenting complaints are shown in Table I.

A large proportion of patients (86\%) had delayed presentation to the tertiary care hospital. Among the reasons for delay in presentation, delayed diagnosis by the medical professionals (primary physician) was in about half $(49 \%, n=25)$ of the patients, $18 \%(n=9)$ of patients had delayed recognition of symptoms by their parents/family, $14 \%(n=7)$ of parents had a delay in seeking medical attention, $4 \%(n=2)$ of parents opted for Hakeem/spiritual treatments earlier, while $2 \% \quad(n=1)$ parents did not seek medical attention due to financial constrains. Under-nutrition was very common with about half of the patients $(51 \%, n=26)$ having weight-for-age less than 5 th percentile and only $8 \%(n=4)$ patients had weight between $50-75^{\text {th }}$ percentile.

Various serious complications were observed in the patients who presented with malignant mediastinal mass. The frequency of various complications is shown in Figure 1. For the definitive diagnosis, tissue biopsy was indicated in $60.7 \%(n=31)$ patients, while the rest of the patients $39.2 \% \quad(n=20)$ were diagnosed on flow cytometry. For the patients in whom tissue biopsy was indicated $(n=31)$, lymph node excision biopsy under general anesthesia was done in $32 \% \quad(n=10)$, thoracostomy under general anesthesia (mediastinal mass biopsy) was done in $29 \%(n=9)$ patients, USGguided mediastinal mass biopsy under local anesthesia and Ketamine sedation was done in $19.4 \%(n=6)$, one patient $(3.2 \%)$ underwent laprotomy under general anesthesia for abdominal lymph node biopsy; whereas in $16 \%(n=5)$ patients, tissue/mass biopsy could not be done due to either unstable condition or expiry before biopsy.

The most common diagnosis was T-cell acute lymphoblastic leukemia (ALL), which was diagnosed in $35 \%$ of the patients $(n=18)$. Second most common diagnoses were lymphoblastic lymphoma (LL) and Hodgkin's lymphoma $(H L)$, both found in $15.7 \%(n=8)$ patients each. Mixed cellularity type was found in six out of 8 patients of $\mathrm{HL}$, while rest two patients had nodular sclerosis type. Neuroblastoma was diagnosed in $7.8 \%$ $(n=4)$ patients. Two patients $(4 \%)$ were diagnosed as acute myeloid leukemia (AML) and non-Hodgkin Lymphoma (NHL type could not be specified) each. One patient of Burkitt lymphoma (2\%) and one of Ewing sarcoma $(2 \%)$ were diagnosed while seven patients $(13.8 \%)$ remained undiagnosed. Majority of the patients $(31.4 \%, n=16)$ presented in stage 3 disease at presentation. Eight patients $(15.6 \%)$ presented with advanced stage (stage 4 ), while only one patient $(2 \%)$ presented in stage 1 disease. Staging in undiagnosed patients could not be done. All patients with acute leukemia; T-cell ALL and AML $(39.2 \%, n=20)$ were categorised as high risk patients rather than in stages. A large proportion of patients $(45 \%, n=23)$ expired during treatment. The frequencies of various complication outcomes are shown in Figure 1.

Various factors were investigated with respect to the outcome. Primary diagnosis was the most significant factor $(p<0.001)$, associated with the outcome of these patients. It was observed that $100 \%(n=2$, total $=2)$ of patients with diagnosis of AML expired, $85 \%(n=6$, total 7) of undiagnosed patients, $44 \%(n=8$, total $=18)$ patients of T-cell ALL, $37.5 \%(n=3$, total $=8)$ patients of Hodgkin-lymphoma and lymphoblastic lymphoma each and $25 \%(n=1$, total $=4)$ of patients with neuroblastoma expired during treatment. Other significant factors were delayed presentation of the patient to the specialised healthcare facility $(p=0.007)$ and educational status of the father/guardian of the patient $(p=0.05)$.

\section{DISCUSSION}

This study showed similar results regarding the median age of presentation and gender distribution in pediatric malignant mediastinal masses to the previously published study from Pakistan. ${ }^{8}$ However, the pattern of clinical presentation, complications and diagnoses varied from other similar studies.1,5,8,12 None of the 
patients were asymptomatic at presentation and fever was the most common symptom (82\%) among all. A large number of our patients presented with serious lifethreatening complications and advanced stage of disease. This may be attributed to delayed referral/ presentation of these patients to the tertiary care hospital. Majority of the patients $(87 \%)$ had delayed presentation and most common reason was delay in diagnosis and referral by the primary physician (49\%).

Regarding the demographic data, majority of patients $(92 \%)$ presented from district Lahore and the nearby districts of the Punjab province. Immunisation status of the patients was also satisfactory. A large number of patients belonged to the lower socio-economic class $(78 \%)$, and majority of the families were uneducated $(39 \%)$. A large percentage of patients were found to be wasted with $51 \%$ patients having weight-for-age less than 5th percentile. A vast majority of the patients with malignant mediastinal mass presented with serious lifethreatening complications (92.2\%). Airway obstruction was the most common complication $(35.3 \%)$ as shown in study by Piestra et al., ${ }^{13}$ but other common lifethreatening complications were pericardial effusion $(19.6 \%)$ superior vena cava syndrome $(13.7 \%)$ and pleural effusion (13.7\%).

In this series of cases, the most common underlying diagnosis was T-cell acute lymphoblastic leukemia $(35.3 \%)$, similar to one study by Freud et al. ${ }^{14}$ It was followed by equal number of cases of T-cell lymphoblastic lymphoma and Hodgkin-lymphoma (15.7\% each). None of our patients were of germ cell tumors. This frequency of underlying diagnoses was different from other previous studies from developing and developed countries. 1,5,7,8,12,14

Many challenges were faced during the management of these patients. In diagnosing mediastinal masses, the procedure of mediastinal mass biopsy under general anesthesia was very challanging, especially in those patients with airway obstruction and as a large proportion of these patients had airway obstruction on presentation. Therefore, many patients were left undiagnosed. Similarly, majority of patients presented with advanced stage of disease and life-threatening disease complications on presentation. Thirty-nine percent $(39 \%)$ patients presented with high risk disease, $16 \%$ with stage 4 disease, and $31.4 \%$ with stage 3 disease; while only $2 \%$ with stage 1 . Similarly $92 \%$ $(n=47)$ patients presented with various life-threatening complications. The management of advanced stage of disease and associated life-threatening complications are always challenging for the oncologists and are associated with increased morbidity and mortality of these patients.

The prognosis of these patients was found to be significantly associated with underlying diagnosis, delayed presentation to the healthcare facility and educational status of head of patient's family. Regarding the association of primary diagnosis, both of two patients of AML who presented with malignant mediastinal mass expired. Moreover, among all expired patients, majority of patients $(34.7 \%$ ) had T-cell ALL, while $26 \%$ of expired patients stayed undiagnosed. Similarly, delayed presentation to the tertiary care facility had prognostic impact as well. Among all the patients who expired, $87 \%$ $(n=20)$ had delayed presentation. The education status of the father/guardian of the patients was also found to be a statistically significant factor for the outcome of those patients. More than half of the patients (56.5\%) who expired belonged to uneducated families, while a very small proportion $(8.7 \%)$ belonged to the families who took education till graduation. Probably, the education status is directly associated with timely recognition of complaints of the patient and seeking medical attention. The education and training of primary care physicians is paramount regarding recognition of signs and symptoms of pediatric oncological diseases and timely referrals to tertiarycare hospitals in order to improve the outcome of these patients.

\section{CONCLUSION}

The spectrum of clinical presentations, complications, and diagnoses of pediatric malignant mediastinal masses is distinctive in this part of the world and varies from international studies. Social problems like poor literacy rate and delay in presentation to healthcare facility not only pose more difficulties and challenges in managing these patients, but also result in higher mortality rates and poor outcomes.

Disclosure: This article was written as a part of requirement for FCPS Pediatric Hematology-Oncology examination.

\section{REFERENCES}

1. Gun F, Erginel B, Unuvar A, Kebudi R, Salman T, Celik A. Mediastinal masses in children: experience with 120 cases. Pediatr Hematol Oncol 2012; 29:141-7.

2. Esposito G. Diagnosis of mediastinal masses and principles of surgical tactics and technique for their treatment. Semin Pediatr Surg 1999; 8:54-60.

3. Cavett C. Mediastinal tumors. In: Ashcraft KW, Ed. Pediatric surgery. Ed. 3rd. Philadelphia: W.B. Saunders, 2000; 318-23.

4. Lerman J. Anterior mediastinal masses in children. Semin Anesth 2007; 26:133-40.

5. Freud E, Ben-Ari J, Schonfeld T, Blumenfeld A, Steinberg R, Dlugy $\mathrm{E}$, et al. Mediastinal tumors in children: $A$ single institution experience. Clin Pediatr (Phila) 2002; 41:219-23.

6. Fisher MJ. Oncologic Emergencies. In: Principles and practice of pediatric oncology. Pizzo PA, Poplack DG (Eds). 6th ed. Philadelphia: Lippincott Williams and Wilkins, 2011; pp 1125.

7. Glick RD, La Quaglia MP. Lymphomas of the anterior mediastinum. Semin Pediatr Surg 1999; 8:69-77.

8. Mushtaq N, Alam MM, Aslam S, Fadoo Z, Haq AU. Malignant 
mediastinal mass in children: A single institutional experience from a developing country. J Pak Med Assoc 2014; 64:386-89.

9. Hammer GB. Anaesthetic management for the child with a mediastinal mass. Paediatr Anaesth 2004; 14: 95-7.

10. Loeffler JS, Leopold KA, Recht A, Weinstein HJ, Tarbell NJ. Emergency prebiopsy radiation for mediastinal masses: impact on subsequent pathologic diagnosis and outcome. J Clin Oncol 1986; 4:716-21.

11. Ferrari LR, Bedford RF. General anesthesia prior to treatment of anterior mediastinal masses in pediatric cancer patients. Anesthesiology 1990; 72: 991-5.
12. Takeda S, Miyoshi S, Akashi A, Ohta M, Minami M, Okumura M, et al. Clinical spectrum of primary mediastinal tumors: A comparison of adult and pediatric populations at a single Japanese institution. J Surg Oncol 2003; 83:24-30.

13. Piastra M, Ruggiero A, Caresta E, Chiaretti A, Pulitano S, Polidori G, et al. Life-threatening presentation of mediastinal neoplasms: report on 7 consecutive pediatric patients. $\mathrm{Am} \mathrm{J}$ Emerg Med 2005; 23:76-82.

14. Tansel T, Onursal E, Dayloglu E, Basaran M, Sungur Z, Camci E, et al. Childhood mediastinal masses in infants and children. Turk J Pediatr 2006; 48:8-12. 